'Unidad de Cirugía Digestiva, Departamento de Cirugía. Hospital Clínico Universidad de Chile, Facultad de Medicina, Universidad de Chile.

2Departamento de Radiología. Hospital Clínico Universidad de Chile, Facultad de Medicina, Universidad de Chile.

${ }^{3}$ Departamento de Anatomía Patológica.

Hospital Clínico Universidad de

Chile, Facultad de Medicina, Universidad de Chile.

Recibido el 10 de mayo de 2013 aceptado el 9 de abril de 2014 .

Correspondencia a:

Dr. Italo Braghetto Miranda

Departamento de Cirugía, Hospital Clínico de la Universidad de Chile.

Santos Dumont 999, Santiago, Chile. cirugía@braghetto.cl

\section{Carcinoma escamoso sarcomatoide de la unión gastroesofágica: caso clínico}

\author{
ITALO BRAGHETTO ${ }^{1}$, GUSTAVO MONTESINOS ${ }^{1}$, MAHER MUSLEH ${ }^{1}$, \\ CLAUDIO CORTÉS ${ }^{2}$, FACUNDO LAS HERAS ${ }^{3}$, CARLOS DOMÍNGUEZ
}

\section{Esophageal sarcomatoid squamous carcinoma. Report of one case}

Sarcomatoid squamous carcinoma (ESC) is a rare esophageal neoplasm, with a clinical, etiological and pathological behavior that differs from squamous cancer. From the histological point of view it has a dual configuration. The squamous epithelial component is usually limited to small areas, while the major part is constituted by mesenchymatous (sarcomatoid) polypoid tissue. Treatment is esophagectomy or total esophagogastrectomy depending on the tumor location. Early detection is critical in terms of survival. For large lesions, preoperative chemo-radiotherapy can be considered. We report a 78-year-old male presenting with dysphagia. An upper gastrointestinal endoscopy showed a tumor located below the cardia. The patient was subjected to a total esophageal and gastric resection. In a second operation, the digestive transit was reconstituted in 2 steps. The pathology report informed a sarcomatoid squamous carcinoma. After 18 months of follow up, the patient is ambulatory.

(Rev Med Chile 2014; 142: 786-790)

Key words: Carcinosarcoma; Esophageal Junction; Esophageal Neoplasms.
E 1 carcinoma escamoso sarcomatoide (CES), descrito en 1865 por Virchow, es una entidad muy poco frecuente en el grupo de neoplasias esofágicas, y pocos casos han sido publicados en la literatura. Esta neoplasia presenta algunas características clínicas, etiológicas y patológicas que determinan un comportamiento diferente al cáncer esofágico escamoso habitual ${ }^{1,2,3}$. Se presenta el caso de un paciente con diagnóstico de CES de la unión gastroesofágica inicialmente diagnosticado como tumor del estroma gastrointestinal (GIST), cuyo tratamiento final consistió en una esofagogastrectomía total y reconstitución en 2 tiempos.

\section{Caso clínico}

Paciente 78 años, sexo masculino. Hipertensión arterial en tratamiento, enfermedad arterial oclusiva carotídea en estudio.
Se presenta con cuadro clínico de 3 meses de baja de peso $9 \mathrm{~kg}$, disfagia y saciedad precoz. Endoscopia digestiva compatible con lesión pediculada subcardial en cara posterior. Biopsia endoscópica: "tumor estromal fusocelular con $>10$ mitosis atípicas por 50 campos", compatible con GIST.

Tomografía axial computada de abdomen: lesión subcardial de $6 \mathrm{~cm}$ diámetro mayor, sin adenopatías ni signos de diseminación sistémica, que se confirma con un tránsito esófago estómago y duodeno con sulfato de bario: lesión pediculada subcardial (Figura 1). Se programa resección tumoral laparoscópica: Gastrotomía anterior, resección de tumor con sutura mecánica a pedículo, gastrorrafia, biopsia de serosa gástrica y peritoneo (Figuras 2 y 3 ). Postoperatorio sin complicaciones.

Biopsia: Carcinoma escamoso fusocelular sarcomatoide, polipoide y ulcerado $6,5 \times 5 \times 4 \mathrm{~cm}$. Margen positivo de pedículo. Sin permeaciones 


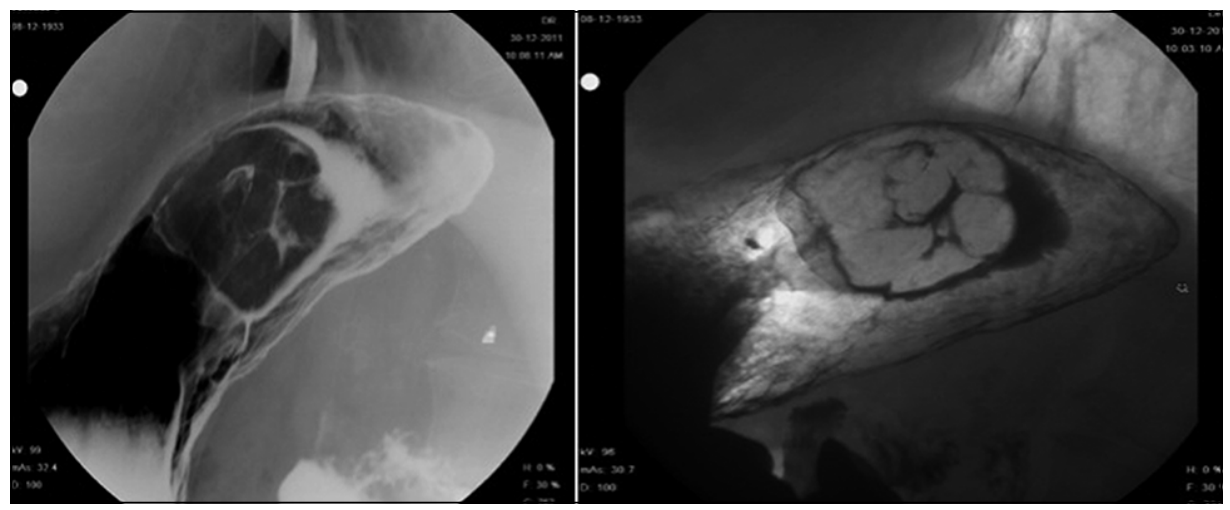

Figura 1. Radiología: lesión pediculada que ocupa región subcardial.

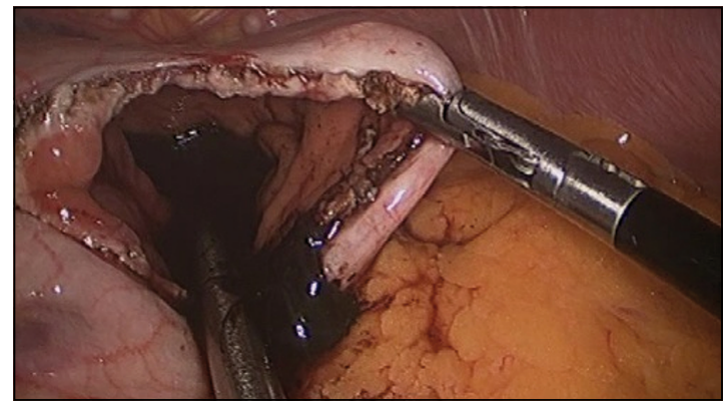

Figura 2. Gastrotomía anterior laparoscópica.

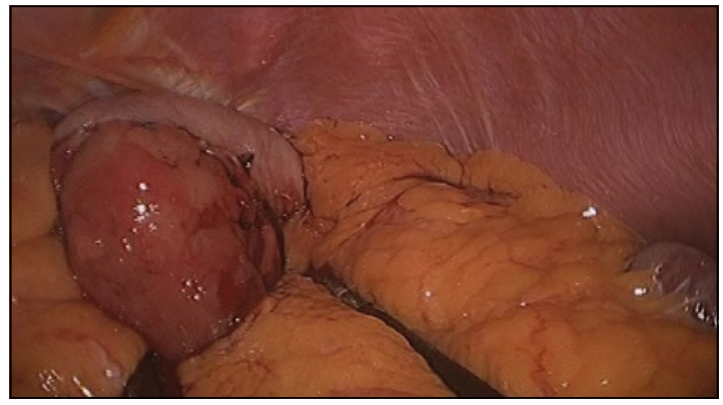

Figura 3. Exposición de tumor.

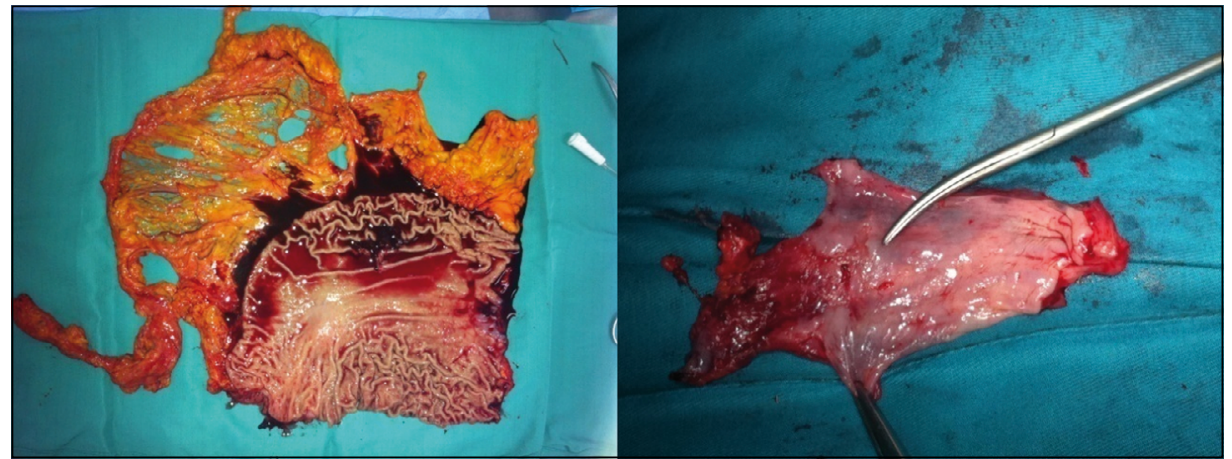

Figura 4. Resección esofagogástrica: compromiso neoplásico de esófago inferior. vasculares, linfáticas ni perineurales. Peritoneo y serosa gástrica: sin neoplasia.

Se programa para gastrectomía total más esofagectomía distal con diagnóstico de carcinoma escamoso de la unión gastroesofágica: en el intraoperatorio se recibió biopsia rápida de límite oral infiltrado por carcinoma, recorte revela compromiso neoplásico proximal por lo que se decidió no reconstituir en un tiempo por las condiciones del paciente en el intraoperatorio, efectuándose esofagogastrectomía total transhiatal (Figura 4), esofagostoma cervical terminal transitorio y yeyunostomía de Wietzel, dejando para un segundo tiempo la reconstitución definitiva del tránsito digestivo.

Biopsia definitiva: Carcinoma espinocelular fusocelular sarcomatoide esofágico con extensión hasta submucosa, compromiso de grupo 1 y 5 

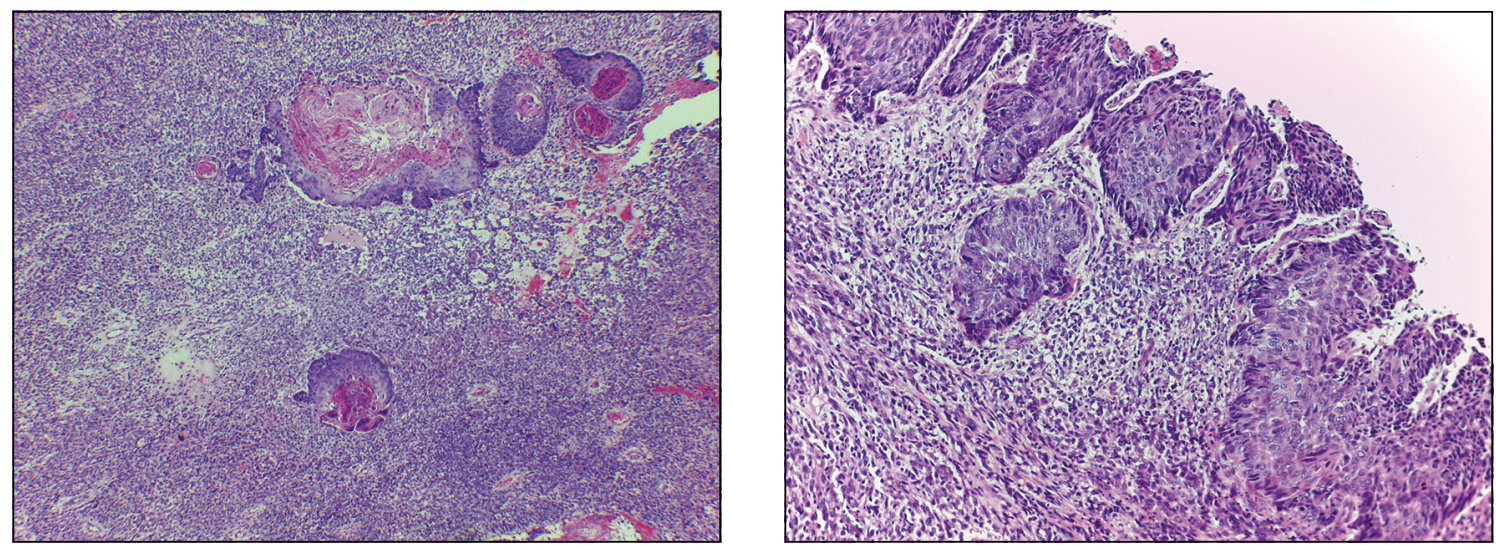

Figura 5. Histología: Hematoxilina eosina, aumento de 100 x, proliferación neoplásica maligna bifásica, de predominio fusiforme, con nidos de carcinoma epidermoide. Pared esofágica revestida por carcinoma escamoso, con proliferación neoplásica fusiforme maligna infiltrante.

(4/37), sin adenopatías mediastínicas. Nódulo metastásico en epiplón menor correspondiente a ganglio $\mathrm{N}^{\circ}$ 5. Márgenes quirúrgicos sin neoplasia (Figura 5).

$\mathrm{Al}$ mes postoperatorio se realizó reconstrucción con ascenso de colon derecho e éleon terminal vía retroesternal: esófago-íleo anastomosis cervical terminolateral, transverso-duodeno anastomosis laterolateral, íleo-transverso anastomosis laterolateral y yeyunostomía de Wietzel.

Evolucionó con fístula cervical de bajo débito sin repercusión clínica, alta al día 21 postoperatorio con régimen líquido y aporte por yeyunostomía.

Paciente en excelentes condiciones a los 18 meses postoperatorios, autovalente, requirió dilatación endoscópica de anastomosis cervical, sin incidentes.

\section{Discusión}

El carcinoma sarcomatoide esofágico fue descrito por Virchow en 1865, actualmente representa entre 0,5 a $2,8 \%$ de las neoplasias esofágicas ${ }^{4}$. Denominado también carcinosarcoma, pseudosarcoma y carcinoma polipoídeo, debido a la incertidumbre en la patogénesis de este tumor, característicamente de configuración polipoídea gruesa, sin embargo, Iascone ${ }^{5}$, después de una revisión de lo publicado, establece que estas entidades no presentan diferencias y que pueden corresponder a un mismo proceso patológico. Desde el punto de vista histológico, presenta una configuración dual: por una parte el componente epitelial, habitualmente escamoso, limitado a pequeñas áreas; mientras que la mayor parte se encuentra constituido por tejido de aspecto mesenquimático (o sarcomatoide) polipoídeo. En el aspecto macroscópico suelen ser tumores polipoídeos voluminosos, de crecimiento rápido, que alcanzan diámetros de hasta $15 \mathrm{~cm}$, con superficie lisa, ulcerada, o símil de mucosa gástrica habitualmente adheridas a la pared por un pedículo, sin embargo, en ocasiones carecen de éste y se encuentran sobre una base de implantación gruesa. La mucosa circundante suele tener aspecto normal. Suelen crecer hacia el lumen, por lo tanto, provocan síntomas precozmente lo que permite su diagnóstico en etapas más precoces ${ }^{6}$.

Al estudio inmunohistoquímico el componente mesenquimático (sarcomatoide) presenta reactividad a desmina, vimentina y actina de músculo liso, con captación focal ocasional con citokeratina. La inmunorreactividad con citokeratina, pancreatina, cromogranina A, CD56 y sinaptofisina es altamente específica para el componente epitelial ${ }^{14}$. La zona transicional entre ambos componentes suele ser reactiva a los mismos elementos que el área mesenquimática.

Respecto a la histogénesis, una teoría sugiere que ambos componentes del tumor se originan de una célula madre, totipotencial (hipótesis divergente) o de una o más células madre (hipótesis 
convergente). También se ha postulado que el componente epitelial representa un estado reactivo no neoplásico en el desarrollo de carcinoma. Hallazgos recientes apoyan la primera teoría y el origen epitelial del componente sarcomatoide vía metaplasia ${ }^{2}$.

El diagnóstico diferencial incluye otras lesiones polipoídeas esofágicas, algunas benignas, como papilomas escamosos y pólipos fibrovasculares o neoplasias malignas como carcinoma escamoso (especialmente el tipo verrucoso). Otras entidades tales como melanoma maligno y sarcomas como histiocitoma maligno fibroso completan la lista de diagnósticos diferenciales ${ }^{5,6,7,8,9}$.

El pronóstico difiere del carcinoma escamoso común, pues tiende a crecer de forma intraluminal más que infiltrando la pared: no suelen invadir más allá de la lámina propia o submucosa. El riesgo de metástasis depende del grado de invasión: $10 \%$ cuando está confinado a lámina propia, $25 \%$ cuando a submucosa y muscular propia y $75 \%$ cuando es transmural. El componente sarcomatoso aparentemente es menos agresivo.

Iascone y Barreca ${ }^{5}$ revisaron casos de carcinosarcoma y otros catalogados como pseudosarcomas para determinar las características clínicas y su pronóstico, no encontrando diferencias entre estos 2 tipos de neoplasia y, por lo tanto, se establece que son el mismo proceso patológico.

Kuo et $\mathrm{al}^{10}$, en una revisión de 3.318 casos de neoplasias esofágicas, 12 correspondieron a carcinosarcoma esofágico, con una tasa de incidencia de 0,36\%, 8 de los 12 fueron del tipo polipoídeo, mientras que 4 del tipo ulcerativo. Al estudio endosonográfico se manifestaron como lesiones hipoecogénicas heterogéneas, con márgenes externos irregulares y componente interno multiquístico.

Las metástasis pueden ser de origen carcinomatoso, sarcomatoide o mixtas, su ubicación habitual son los linfonodos regionales, seguidos por pulmón y pleura ${ }^{11,12,13}$.

El tratamiento suele ser la esofagogastrectomía total, siendo la detección precoz fundamental en términos de sobrevida. Para grandes lesiones puede considerarse la quimiorradioterapia preoperatoria. Se han descrito también casos de carcinosarcoma a nivel gástrico, tal como inicialmente fue planteado en el caso presentado en este artículo $^{6,9,11,14}$.

En relación a la sobrevida, se ha descrito que a 3 años es superior al carcinoma escamoso (63\% vs $28 \%$ ), en parte por su capacidad de crecimiento intraluminal que los hace más sintomáticos, lo que determina una menor tasa de compromiso linfonodal (al ser detectados en etapas precoces); sin embargo, a 5 años de seguimiento, esta diferencia no es significativa ( $27 \%$ vs $22 \%)$. Es importante notar que estos datos provienen de la única serie de casos publicada (20 pacientes) por Iyomasa et al. ${ }^{8,9,10,12}$.

\section{Conclusión}

Si bien es una patología infrecuente, el carcinoma escamoso sarcomatoide debe ser considerado en el diagnóstico diferencial de todo tumor de la unión gastroesofágica. En ese sentido, este caso muestra características distintivas en lo endoscópico-quirúrgico (como tamaño y aspecto pediculado) y elementos histopatológicos distintivos que orientan hacia su sospecha diagnóstica. Su mejor sobrevida a 3 años respecto al cáncer escamoso clásico se debe, fundamentalmente, a la detección precoz debida al crecimiento intraluminal de esta neoplasia. Concordamos con la literatura en que su tratamiento óptimo es la esofagogastrectomía total.

\section{Referencias}

1. Iezzoni JC, Mills SE. Sarcomatoid carcinomas (carcinosarcomas) of the gastrointestinal tract: a review. Semin Diagn Pathol 1993; 10: 176-87.

2. Szumiło J, Dabrowski A, Zinkiewicz K, Chyzy ska M, Korobowicz E. Sarcomatoid carcinoma of the esophagus. Pol J Pathol 2005; 56: 47-50.

3. Ohtaka M, Kumasaka T, Nobukawa B, Hirai S, Suda K, Ohno Y, et al. Carcinosarcoma of the esophagus characterized by myoepithelial and ductal differentiations. Pathol Int 2002; 52: 657-63.

4. Oak JH, Chung WC, Jung JH, Kim JD, Lee JR, Paik CN, et al. A case of carcinosarcoma in a patient with corrosive esophagitis. Korean J Gastroenterol 2008; 52: 42-7.

5. Iascone C, Barreca M. Carcinosarcoma and pseudosarcoma of the esophagus: two names, one diseasecomprehensive review of the literature. World J Surg 1999; 23: 153-7.

6. Solerio D, Ruffini E, Camandona M, Raggio E, Castella- 
no I, Dei Poli M. Carcinosarcoma of the esophagogastric junction. Tumori 2008; 94: 416-8.

7. Tomizawa Y, Taniguchi M, Mori M. An unusual case of intraluminally growing esophageal tumor. Diagnosis: Carcinosarcoma of the esophagus. Gastroenterology 2011; 141: e10-1.

8. Zhao S, Xue Q, Ye B, Lu H, He J, Zhao H. Synchronous primary carcinosarcoma and adenosquamous carcinoma of the esophagus. Ann Thorac Surg 2011; 91: 926-8.

9. Kuo CJ, Lin TN, Lin CJ, Wu RC, Chang HK, Chu YY, et al. Clinical manifestation of esophageal carcinosarcoma: a Taiwan experience. Dis Esophagus 2010; 23: 122-7.

10. Raza MA, Mazzara PF. Sarcomatoid Carcinoma of
Esophagus. Arch Pathol Lab Med 2011; 135: 945-8.

11. Kikuyama R, Tanaka K, Tano S, Iguchi T, Nishikawa K, Harada T, et al. A case of gastric carcinosarcoma. Endoscopy 2009; 41: E220.

12. Iyomasa $S$, Kato $H$, Tachimori $Y$, Watanabe $H$, Yamaguchi H, Itabashi M. Carcinosarcoma of the esophagus: A twenty-case study. Jpn J Clin Oncol 1990; 20: 99-106.

13. Madan AK, Long AE, Weldon CB, Jaffe BM. Esophageal carcinosarcoma. J Gastrointest Surg 2001; 5: 414-7.

14. Randjelovic T, Filipovic B, Babic D, Cemerikic V, Filipovic B. Carcinosarcoma of the stomach: A case report and review of the literature. World J Gastroenterol 2007; 13: 5533-6. 